\title{
Enhancement of Optical, Thermal and Mechanical Properties of Kap Crystals by Magnesium Doping
}

\author{
V. Thendral ${ }^{*}$, A. Thayumanavan ${ }^{\mathrm{G} 1}, \mathrm{G}$. Pasupathi ${ }^{1}$, G. Marudhu ${ }^{2}$ \\ ${ }^{1}$ PG and Research Department of Physics, A.V.V.M Sri Pushpam College, Poondi-613503, Thanjavur, \\ Tamilnadu, India. \\ ${ }^{2}$ Department of Physics, B.S. Abdur Rahman University, Chennai - 600 048, India.
}

\begin{abstract}
The effect of dopant $\mathrm{Mg}^{2+}$ on the growth process, crystalline properties of potassium acid phthalate $(K A P)$ are investigated. Optically transparent single crystals were grown by a slow evaporation solution growth technique at a room temperature has been investigated. Single crystal XRD analysis reveals slight changes in the lattice parameters as a result of doping. The modes of vibration in the crystal lattice has been determined by FTIR analysis. The UV-Vis-NIR spectra revealed the transmission properties, thermal absorption properties of the pure and doped KAP crystals were investigated using thermo gravimetric analysis (TGA) and differential thermal analyses (DTA), Mechanical properties were studied using vickers microhardness tester. The doping result in morphological changes and significantly improves the second harmonic generation (SHG) efficiency of the host crystal.
\end{abstract}

\section{Introduction:}

Potassium hydrogen phthalate (KAP) crystal is well known for its application in the production of crystal analyser for long wave X-ray spectrometer [1,2]. KAP belongs to the series of alkali acid phthalates which crystallizes in the orthorhombic structure. The crystals have excellent physical properties and have a good record for long term stability in devices [3-5]. KAP possesses piezo-electric pyro-electric, elastic and nonlinear optical properties [6-10]. KAP crystals are the promising candidates for the qualitative and quantitative X-ray spectral analysis of light elements such as $\mathrm{Fe}, \mathrm{Al}, \mathrm{Mg}, \mathrm{F}$ and $\mathrm{Si}$ in a long wave spectral area and act as good monochromators $[11,12]$.

The influence of impurities on the habit morphology [13-16], physical, chemical and mechanical properties of KAP single crystals were studied [17-19]. Motivated by these considerations, single crystals of pure, $\mathrm{Mg}^{2+}$ doped KAP crystals are grown in aqueous solution by slow evaporation technique. Thus the grown crystals are subjected to various characterizations such as thermal, optical, mechanical etc., are discussed in detail.

\section{Crystal Growth:}

Pure KAP was purified by successive recrystallization. The crystals were grown by a slow evaporation solution growth technique. A saturated aqueous solution of KAP was prepared. Magnesium nitrate was taken as dopants with $1 \%$ concentration by weight in the mother solution. The solution was filtered with a microfilter. The seed crystals are allowed to float on the surface of the saturated solution and left for slow evaporation at room temperature $\left(30^{\circ} \mathrm{C}\right)$. The crystallization took place within 10-15 days and the optically transparent crystals of metal ions doped KAP were obtained with larger dimensions.

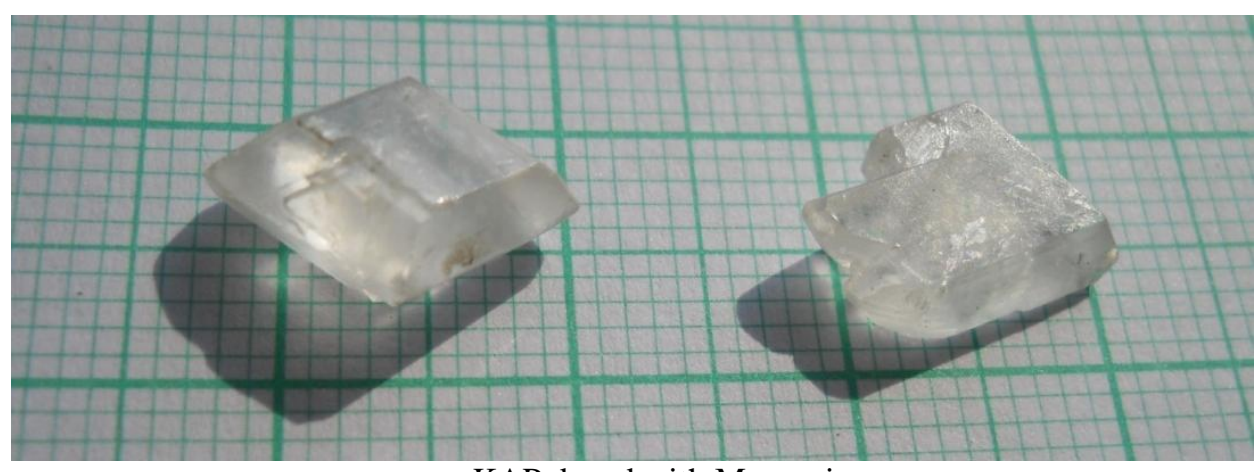

KAP doped with Magnesium

Fig. 1. 


\subsection{Single crystal $X$-ray diffraction}

\section{Result and Discussion}

The single crystal X-ray diffraction analysis on $\mathrm{Mg}^{2+}$ doped potassium acid phthalate single crystals (KAP) was recorded using ENRAF NONIUS CAD 4 automatic X-ray diffractonmeter. This analysis has revealed that the single crystals of doped KAP crystallize in orthorhombic system with space group Pca. The calculated lattice parameters for magnesium doped KAP crystals are compared with that of pure KAP [20] and are given in table 1. The XRD analyses revealed that the addition of metal ion impurities in the KAP crystal does not change the crystal structure though there is a small change in the lattice parameters.

Table 1: Comparison of unit cell parameters

\begin{tabular}{|l|l|l|}
\hline & \multicolumn{1}{|c|}{ Pure KAP } & \multicolumn{1}{c|}{ Magnesium doped KAP } \\
\hline $\mathrm{a}\left(\mathrm{A}^{\mathrm{o}}\right)$ & $9.60 \mathrm{~A}^{\mathrm{o}}$ & $9.62 \pm 0.01 \mathrm{~A}^{\mathrm{o}}$ \\
\hline $\mathrm{b}\left(\mathrm{A}^{\mathrm{o}}\right)$ & $6.46 \mathrm{~A}^{\mathrm{o}}$ & $6.47 \pm 0.071 \mathrm{~A}^{\mathrm{o}}$ \\
\hline $\mathrm{c}\left(\mathrm{A}^{\mathrm{o}}\right)$ & $13.85 \mathrm{~A}^{\mathrm{o}}$ & $13.308 \pm 0.02 \mathrm{~A}^{\mathrm{o}}$ \\
\hline System & Orthorhombic & Orthorhombic \\
\hline Space group & Pca & Pca \\
\hline Volume $\left(\mathrm{A}^{\mathrm{o}}\right)^{3}$ & $858.921 \mathrm{~A}^{03}$ & $829 \pm 1.4 \mathrm{~A}^{03}$ \\
\hline
\end{tabular}

\subsection{FTIR Analysis}

The FTIR spectra for pure as well as doped KAP crystals were recorded using an AVATAR 330 FT-IR instrument using the $\mathrm{KBr}$ pellet technique in the range $400-4000 \mathrm{~cm}^{-1}$. The calculated frequencies are agree with the observed frequencies. The frequencies with their relative intensities obtained in FTIR of pure and doped KAP and their most probable assignment are presented in table 2.

Table 2: Vibrational frequencies obtained for pure and doped KAP crystals

\begin{tabular}{|c|c|l|}
\hline \multicolumn{2}{|c|}{ Observed IR frequencies } & \multirow{2}{*}{ Assignments } \\
\hline Pure KAP & Mg doped KAP & \\
\hline 3750 & - & O-H asymmetrical stretching \\
\hline 3426 & 3429 & O-H stretching Hydrogen bond \\
\hline 2479 & 2477 & C-H aromatic Stretching \\
\hline 1950 & 1943 & =C-H out of plane bending \\
\hline 1673 & 1670 & C=C Stretching \\
\hline 1562 & 1567 & $\begin{array}{l}\text {-C=O Carboxylate ion =Oasymmetric } \\
\text { stretching }\end{array}$ \\
\hline 1481 & - & C=C ring stretching \\
\hline 1380 & 1374 & $\begin{array}{l}\text {-C=O } \text { carboxylate ion } \\
\text { =O symmetric stretching }\end{array}$ \\
\hline 1284 & 1278 & $\begin{array}{l}\text {-C=O carboxylate ion } \\
=\text { O symmetric stretching }\end{array}$ \\
\hline & & C-C stretching \\
\hline 1147 & - & C-C-O stretching \\
\hline 1089 & 1092 & C-H out of plane bending \\
\hline 848 & - & C-C stretching \\
\hline 762 & - & =C-H out of plane deformation \\
\hline 717 & - & C-O wagging \\
\hline 684 & 694 & C-C-C out of plane ring deformation \\
\hline 578 & - & C-C-C out of plane ring deformation \\
\hline 548 & 553 & C=C out of plane ring bending \\
\hline 442 & - & C=C out of plane bending \\
\hline 412 & - &
\end{tabular}


FTIR Spectrum
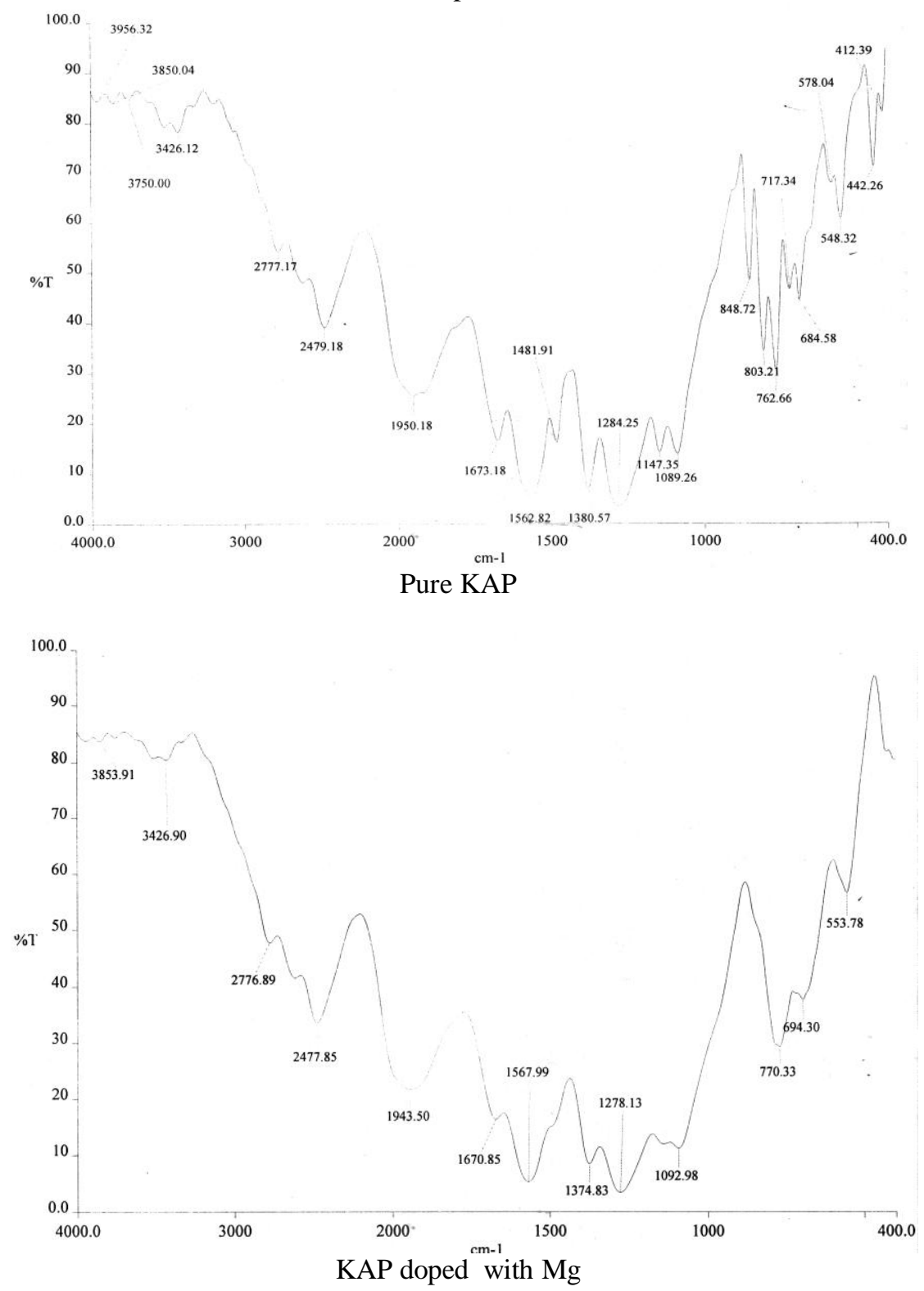

Fig.2.

In all the spectra, the characteristic $\mathrm{OH}$ stretching peaks at $3750 \mathrm{~cm}^{-1}$ in KAP is disappear in magnesium doped KAP and also the peak at 3426 is shifted to 3429 in Mg doped KAP(MKAP) The asymmetric stretching vibration of the carboxylate ion is shifted to higher energy $1567 \mathrm{~cm}^{-1}$ for MKAP respectively compared to pure KAP $\left(1560 \mathrm{~cm}^{-1}\right)$. In addition the $\mathrm{c}=\mathrm{c}$ stretching vibration remains the same at $1671 \mathrm{~cm}^{-1}$. The variation in the FTIR spectrum for the doped crystals is due to the incorporation of metal ions. The spectrum of pure and magnesium doped KAP is shown in Fig. 2.

\subsection{Optical Transmission Studies}

UV-visible spectral study is a useful tool to determine the transparency, which is an important requirement for a material to be optically active [21]. The optical transmission spectrum of the pure and metal ion doped KAP single crystals was recorded in the range of 190-1100 nm using Lambda 35 spectrometer (Fig 3). 


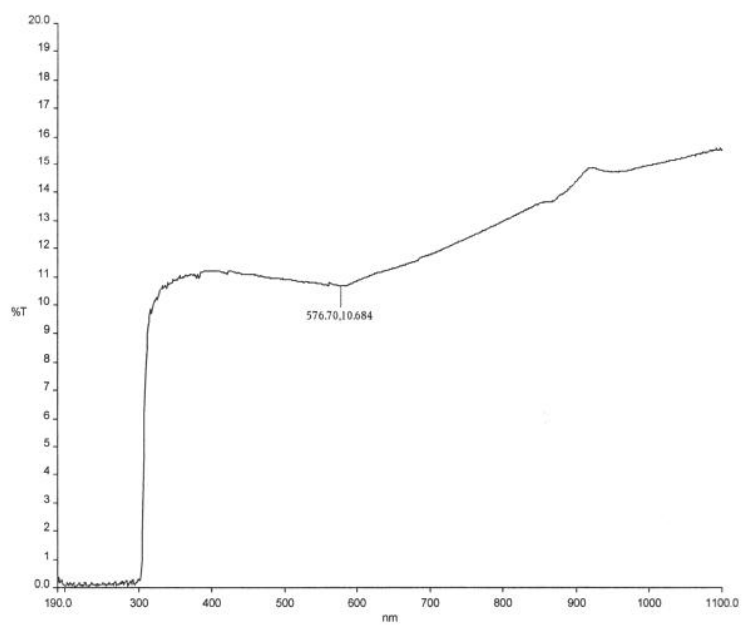

Fig. 3. Transmission spectrum of magnesium doped KAP crystals.

A large absorption was found at $300 \mathrm{~nm}$ and the crystals have very low absorbance in the entire visible and IR region. The UV spectrum reveals that the cut off wavelength is $\sim 300 \mathrm{~nm}$ and a wide transparency in the entire visible region which makes the material suitable for second harmonic generation.

The energy dependence of the absorption coefficient suggests the occurrence of direct band gap and hence it obeys the relation for high photon energy.

$$
(\alpha h v)^{2}=A(h v-E g)
$$

Where Eg is the optical band gap and A is a constant. The variation of $(\alpha h v)^{2}$ vs $h v$ in the fundamental absorption region is plotted in Fig 4. and Eg is evaluated by the Extrapolation of the linear part. The band gap is found to be $3.9 \mathrm{eV}$ for magnesium doped KAP crystals

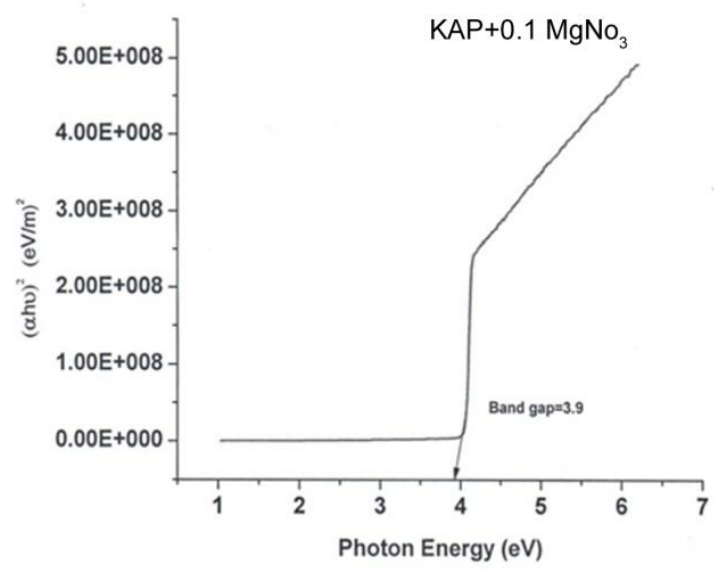

Fig. 4.

\subsection{Microhardness}

Measurement of hardness is a useful nondestructive testing method to determine the hardness of the material. The hardness of a material depends on different parameters such as lattice energy, Debye temperature, heat of formation and interatomic spacing $[22,23$.$] . The microhardness value correlates with other mechanic$ properties such as elastic constants and yield strength. According to Gong [24], during an indentation process, the external work applied by the indenter is converted into a strain energy component which is proportional to the volume of the resultant impression and a surface energy component found to be proportional to the area of the resultant impression. Vickers microhardness test was carried out on pure KAP and metal ions doped KAP single crystals using microhardness tester flitted with a diamond indenter. The indentations were made using a Vickers pyramidal indenter for various loads from 25, 50 and 100g. The Vickers microhardness number (Hv) was evaluated from the relation. 


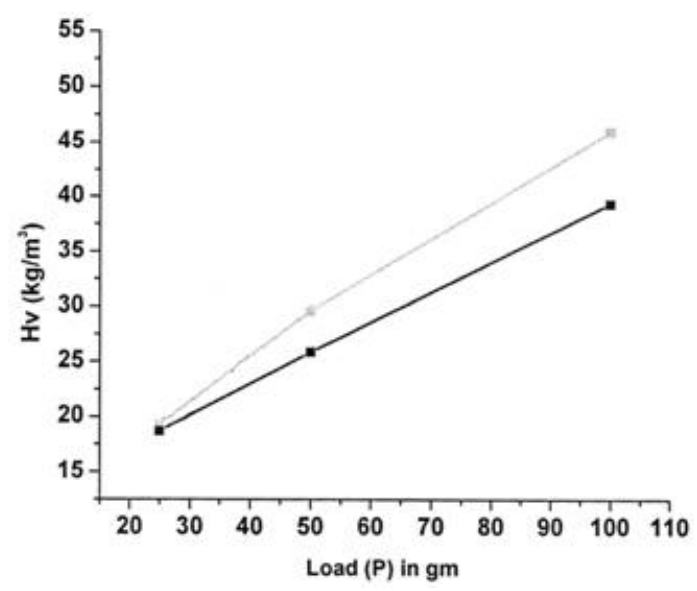

Fig. 5:

$\mathrm{H}_{\mathrm{v}}=1.8544\left(\mathrm{p} / \mathrm{d}^{2}\right) \mathrm{kg} / \mathrm{mm}^{2}$, where $\mathrm{p}$ is the applied load in $\mathrm{g}$ and $\mathrm{d}$ is the diagonal length of the impression in $\mathrm{mm}$.

The variation of microhardness values with applied load is shown in Fig5.

From the vicker's microhardness studies, it is observed that the hardness value increases upto a load of $100 \mathrm{~g}$. At slightly higher loads significant cracking occurs, which may be due to the release of internal stress generated by indentation. The presence of cracks confirms the decrease in microhardness [25]. Onitsch [26] inferred that for hard materials the value $\mathrm{n}$ (work hardening index) lies between 1 and 1.6 and for soft materials it is above 1.6. The work hardening coefficient $n$, a measure of the strength of the crystal is completed from the $\log \mathrm{p}-\log \mathrm{d}$ plot (Fig 6) and it is found to be 1.76 for pure KAP, and 1.86 for magnesium doped KAP. Hence, it is concluded that pure and metal ions doped KAP belongs to soft materials.

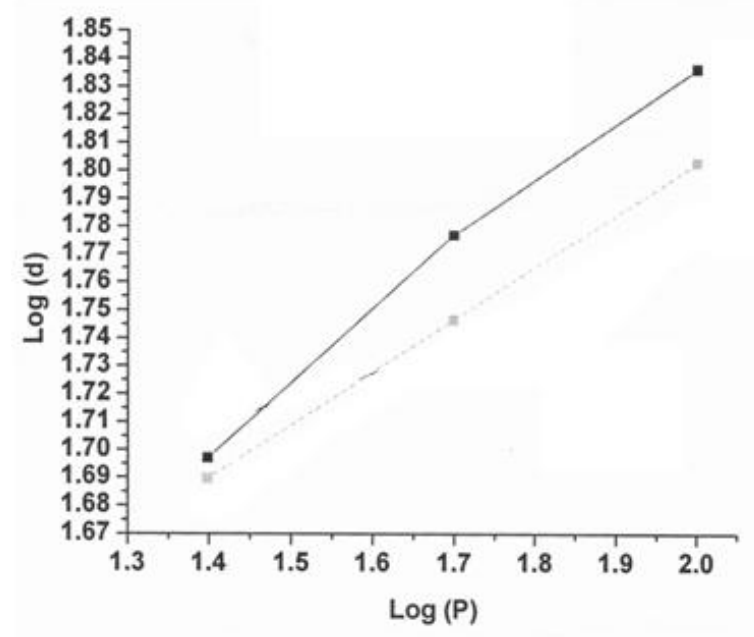

\subsection{Nonlinear Optical Studies}

Fig. 6:

The second harmonic generation Efficiency measurement test on the pure and doped KAP crystals was performed by the kurtz powder SHG method (27). This technique remains an extremely valuable tool for initial screening of materials for second harmonic generation. The fundamental beam of wavelength $1064 \mathrm{~nm}$ from Qswitched Nd:YAG laser was used to test SHG property of the pure and magnesium doped KAP. Pulse energy of $4 \mathrm{~mJ} /$ pulse, pulse width of $8 \mathrm{~ns}$ and repetition rate of $10 \mathrm{HZ}$ was used in both measurement. The fundamental beam was filtered using an IR filter and photomultiplier tube (Philips photonics) was used as the detector. The emission of green light confirms the second harmonic generation on KAP as well as magnesium doped KAP crystals. The SHG efficiency of magnesium doped KAP is roughly 0.83 times that of KDP respectively.

The efficient SHG demands specific molecular alignment of the crystal to be achieved facilitating nonlinearity in the presence of a dopant. It has been reported that the SHG can be greatly enhanced by attaining the molecular alignment through inclusions complexation [28]. Recently, the enhancement in crystalline perfection could lead to improvement in SHG efficiency [29]. 


\subsection{Thermal (TG-DTA) analysis:}

Thermogram provides information about decomposition patterns of materials and weight loss [30]. Simultaneous TG-DTA-DSC curves were recorded on a SDT Q 600 (TA instrument) thermal analyzer. The TGDTA curves were simultaneously obtained in nitrogen at a heating rate of $10^{\circ} \mathrm{c} \mathrm{min}^{-1}$ (Fig. 7). This curve reveal that there is no physically observed water in the molecular structure of crystals grown from magnesium - added KAP solution.

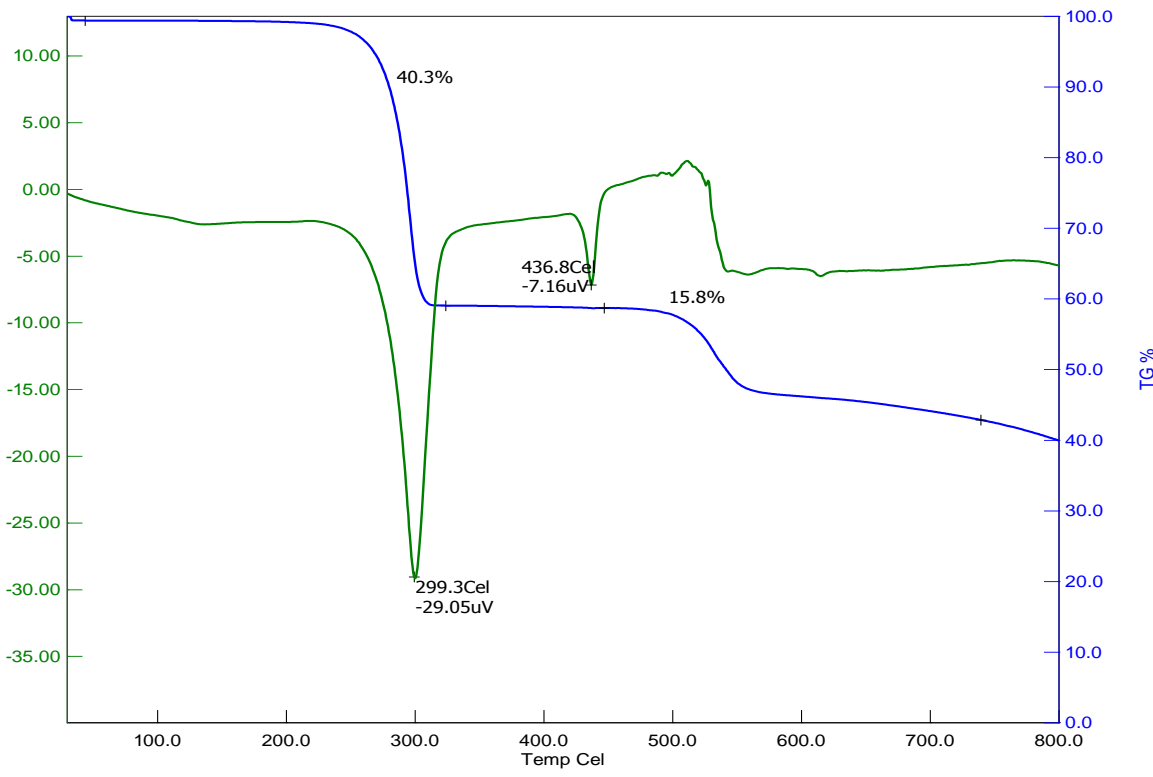

Fig. 7.

The TG curve provides with a quantitative measurement of mass change associated with the transition. It indicates that on melting the material decomposes and loses mass.

There are two weight losses of $40.3 \%$ and $15.8 \%$ noted in the thermogram. Thus the curve shows a gradual mass loss. From the DTA curve it is observed that, the material is stable up to $299^{\circ} \mathrm{c}$, the melting point of the substance. Above this point, the material begins to attain an endothermic transition and begins to decompose. The endothermic peak is obtained in DTA for doped KAP crystals at a little bit higher temperature $\left(299^{\circ} \mathrm{c}\right)$ [31]. The sharpness of this endothermic peak shows the good degree of crystallinity of the sample [32].

\section{Conclusions:}

Good optical quality single crystals of magnesium doped potassium acid phthalate (KAP) have been grown from aqueous solution by slow evaporation technique under room temperature. The metal ions doped crystals were characterized by single crystal X-ray diffraction and confirmed that the crystals belong to orthorhombic system. The presence of all the functional groups and the presence of dopants were determined qualitatively using FTIR analysis. The optical transmission studies revealed that metal ion doped KAP crystals have low absorption in the entire visible region and uv cut-off wavelength was found at 300nm for the doped KAP crystals. TG-DTA studies reveal the suitability of the material for laser applications. The microhardness values of doped KAP crystals are found to be increased by the presence of magnesium dopants. With promising structural, optical and mechanical properties, of magnesium doped KAP single crystals are a potential material for frequency conversion device applications.

\section{Reference}

[1] Jones JL, Paschen KW, Nicholson JB, J Appl Opt. 2(1963) 955

[2] Yoda O, Miyashita A, Murakami K, Aoki S, Yamaguchi N. Proc SPIE Int Soc Opt Eng. 1503(1991) 463

[3] L. Beck, P. Stemmler, and F. Legrand, Rev. Sci. Instr. 66 (1995) 1601.

[4] Jason B. Benedict, Paul M. Wallace, Philip J. Reid, Sei-Hum Jang, and B. Kahr, Adv. Mater. 15 (2003) 1068.

[5] N. Kejalakshmy and k. Srinivasan, J. Phys. D; Appl. Phys. 36 (2003) 1778.

[6] A. Miniewicz, S. Bartkiewicz, Adv. Mater. Opt. Electron. 2 (1993) 157.

[7] F. Kajzar, A. Lorin J. Le Moigne, J. Szpunar, J. Acta Phys. Pol. A 87 (1995) 713.

[8] M.V. Shankar, K.B.R. Varma, Ferro electrics Lett. 21 (1996) 55.

[9] G.R. Ester, P.J. Halfpenny, J. Cryst. Growth 187 (1998) 111.

[10] N. Kejalakshmy, K. Srinivasan, Opt. Mater 27 (2004) 389.

[11] Zhao shujun, Xu Jihua, Xiang Zhilin, Wang Web hao, Nucl. Fusion plasma phys. 13 (1993) 61.

[12] K. Yamashita, N. Watanabe, O. Mastsuvo, J. Yamazaki, I. Hatsukade, T. Ishigami, S. Takahama, K. Tamura, M. Ohtani, Rev. Sci. Instr. 63 (1992) 1217. 
[13]

[28] Rak M, Eremin NN, Eremina TA, Kuznetsov VA, Okhrimenko TM, Furmanova NG, et al. J. Cryst Growth. 273 (2005) 577.

[29] Wang Y, Eaton DF. Chem Phys Lett. 120 (1985) 441.

[30] C.M. Earnet, Anal. Chem . 59 (1984) 1471-1475.

[31] M. Senthil Pandian, N. Balamurugan, G. Bhagavannarayana and P.Ramasamy, J. Cryst. Growth 310. (2008) 4143.

[32] Y. Le Fur, R. Masse, M.Z. Cherkaoui, J. F. Nicoud, kristallogr. Z. 210 (1995) 856 - 860.

K. Sangwal, K. Wojcik, and J. Borc, Cryst. Res. Technol. 38 (2003) 684.

Jason B, Benedict, Theresa Bullard, Werner Kaminsky, and Bart Kahr, Acta cryst. C 60 (2004) 551.

.

(2010) $861-865$

. Mohan Kumar, D. Rajan Babu, P. Murugakoothan, R. Jayavel. J. Cryst. Growth 245 (2002) 297.

R. Uthrakumar, C. Vesta, C. Justin Raj, S. Krishnan, S. Jerome Das, Curr. Appl. Phys. 10 (2010) 548 - 552.

J. Gong, J. Mater, Sci. Lett. 19 (2005) 515 - 517.

C. Hays, E.G. Kendall, Metallography 6 (1973) 275-282.

E.M. Onitsch, Microkope 95 (1950) 12-14. 